\title{
Hepatitis B virus promotes autophagic degradation but not replication in autophagosome
}

\author{
Huayu Yang ${ }^{1, * * *}$, Qining $\mathrm{Fu}^{1,2, *}$, Chen $\mathrm{Liu}^{1}$, Taisheng $\mathrm{Li}^{3}$, Yanan Wang ${ }^{4}$, Hongbing Zhang ${ }^{4}$, \\ Xin Lu ${ }^{1}$, Xinting Sang ${ }^{1}$, Shouxian Zhong ${ }^{1}$, Jiefu Huang ${ }^{1}$, Yilei Mao ${ }^{1, * *}$ \\ ${ }^{1}$ Department of Liver Surgery, Peking Union Medical College Hospital, Chinese Academy of Medical Sciences, Beijing, China; \\ ${ }^{2}$ Department of Vascular Surgery, the First Affiliated Hospital of Chongqing Medical University, Chongqing, China; \\ ${ }^{3}$ Department of Infectious Diseases, Peking Union Medical College Hospital, Chinese Academy of Medical Sciences, Beijing, China; \\ ${ }^{4}$ State Key Laboratory of Medical Molecular Biology, Department of Physiology, Institute of Basic Medical Sciences and School \\ of Basic Medicine, Peking Union Medical College, Chinese Academy of Medical Sciences, Beijing, China.
}

\begin{abstract}
Summary
In this study, we investigate the relationship of hepatitis B virus (HBV) infection and autophagy. HepG2 cells and HepG2 cells infected with HBV (HepG2.2.15) were transfected with GFP-LC3 (green fluorescence protein conjugated with microtubuleassociated protein 1 light chain 3 ) expression vector and autophagy status was then examined with confocal microscope. HepG2.2.15 cells were further treated with serum-free medium or 3-methyladenine (3-MA), and subjected to Hepatitis B core antigen (HBcAg), Hepatitis B surface antigen (HBsAg), or hepatitis B polymerase protein detection by immunohistochemistry. Localization of the GFP-LC3 and the HBV proteins was observed by confocal fluorescence microscope. The level of SQSTM1/p62 protein was also evaluated by Western blot analysis. In contrast to a diffuse distribution in HepG2 cells, GFP-LC3 formed distinct punctate dots, which were further enhanced by nutritional starvation, in HepG2.2.15 cells. The expression of hepatitis B polymerase and $\mathrm{HBcAg}$, but not $\mathrm{HBsAg}$, was positively correlated with the autophagic intensity. However, no co-localizations were observed between HBV proteins and autophagosomes. Suppression of autophagy reduced the expression of hepatitis B polymerase and HBcAg, but not HBsAg. Western blot showed that SQSTM1/p62 protein level was declined in HepG2.2.15 cells comparing HepG2 cells, and further reduced while upon serum starvation. In conclusion, HBV infection induces autophagic degradation and autophagy. Autophagy is critical for HBV replication. However HBV replication does not take place in autophagosomes.
\end{abstract}

Keywords: Hepatitis B virus, autophagy, virus replication, HepG2 cell

\section{Introduction}

Hepatocellular carcinoma (HCC) is one of the most malignant cancers worldwide, ranking the $5^{\text {th }}$ highest in morbidity and the $3^{\text {rd }}$ highest in mortality among

\footnotetext{
*These authors contributed equally to this works.

**Address correspondence to:

Dr. Yilei Mao, Department of Liver Surgery, Peking Union Medical College Hospital, \# 1 Shuai-Fu-Yuan, Wang-Fu-Jing, Beijing 100730, China.

E-mail: yileimao@126.com
}

Dr. Huayu Yang, Department of Liver Surgery, Peking Union Medical College Hospital, 1\# Shuai-Fu-Yuan, Wang-Fu-Jing, Beijing 100730, China.

E-mail: dolphinyahy@hotmail.com various cancers (1). Chronic hepatitis B virus (HBV) or hepatitis $\mathrm{C}$ virus ( $\mathrm{HCV}$ ) infection is found to be associated with nearly $80 \%$ of $\mathrm{HCC}$ (2). $\mathrm{HCC}$ is particularly troublesome in China since over 55\% new HCC cases worldwide were discovered in China, strongly due to the presence of 93 million HBV carriers $(3,4)$. However, the pathogenesis of HBV and its underlying mechanisms for carcinogenesis is not well understood. Thus, this lack of understanding hinders the effective therapeutic approaches for curing HBV infections or decreasing its contribution to carcinogenesis.

Autophagy is a conserved cellular process in which double-membraned vesicles are formed, containing excess or dysfunctional proteins as well as cellular 
organelles, and delivered to lysosomal machinery for degradation. It is a highly regulated process that is essential for maintaining cellular homeostasis in response to multiple stress signals, including nutrient starvation, growth factor deprivation, genotoxic stress, and hypoxia. Autophagy is also known as one of the host defense responses against infections. It has been shown that autophagy is involved in selectively targeting intracellular pathogens, leading to their direct elimination (5-9). It also plays a role in the MHC class II antigen presenting for foreign antigens (10). Thus, some bacteria and viruses developed strategies to suppress or bypass cellular autophagy to ensure their survival (11). Some viruses, such as poliovirus, rhinoviruses, mouse hepatitis virus, SARS-CoV, even hijacked the components of autophagic pathway to facilitate their own replication $(12,13)$.

It has been demonstrated that HBV induces autophagy and uses it to facilitate its DNA replication. This process is mediated by $\mathrm{HBV} \mathrm{X}(\mathrm{HBx})$ protein, which binds to and activates class III phosphatidylinositol-3-kinase (C3PI3K), an enzyme important for autophagy initiation (14). There is also evidence showing that HBx protein can up-regulate the expression of Beclin 1, the mammalian orthologue of the yeast autophagy protein Apg6 (15). However, the mechanism of how the autophagy contributes to viral DNA replication remains unclear.

In this study, we explored the role of autophagosomes in HBV replication by observation of autophagosomes and HBV with a confocal microscope. Meanwhile, SQSTM1/p62, one of the proteins involved in autophagy, was analyzed by western blotting for further understanding the function of autophagy.

\section{Materials and Methods}

\subsection{Strain, plasmid, and cell lines}

Competent Escherichia coli strain DH5 $\alpha$ was purchased from Transgen Biotech Corp. (Beijing, China). Expression plasmid pEGFP-C1-LC3 and the HepG2 cell line were kindly provided by Prof. Hongbing Zhang. The HepG2.2.15 cell line was obtained from Peking University Hepatology Institute, which was transfected with HBV genome and thus stably express HBV proteins and produce virions.

\subsection{Cell culture and transfection}

Both HepG2 and HepG2.2.15 cells were maintained in DMEM (Dulbecco's Modified Eagle Medium) supplemented with $10 \%$ fetal calf serum, 100 units/ $\mathrm{mL}$ penicillin, and $100 \mathrm{mg} / \mathrm{mL}$ streptomycin. The HepG2.2.15 cultural medium has additions of G418 (Wako, Japan) for a final concentration of $380 \mu \mathrm{g} / \mathrm{mL}$. The pEGFP-C1-LC3 transfection was performed using Lipofectamine 2000 (Invitrogen, CA, USA).

\subsection{Immunofluorescence}

HepG2.2.15 cells plated on coverslips were washed with PBS and fixed with $4 \%(\mathrm{v} / \mathrm{v})$ formaldehyde in PBS for 10 min at room temperature. Cells were then permeabilized with $0.2 \%$ Triton X-100 in PBS for 10 $\mathrm{min}$ at room temperature followed by blocking in TBS-T solution $(25 \mathrm{mM}$ Tris, $\mathrm{pH}$ 7.4, $3.0 \mathrm{mM} \mathrm{KCl}, 140 \mathrm{mM}$ $\mathrm{NaCl}$ and $0.05 \%$ Tween 20$)$ containing $5 \%(\mathrm{w} / \mathrm{v})$ BSA (FirstLink, UK) for $30 \mathrm{~min}$. Cells were incubated with one of the primary antibodies, anti-HBcAg (Abcam, CA, USA), anti-HBsAg (Abcam), or anti-hepatitis B polymerase antibody (Santa Cruz Biotechnology, CA, USA) at $4^{\circ} \mathrm{C}$ overnight. Goat anti-mouse IgG antibody conjugated to Сy3 (Beyotime Institute of Biotech, China) was used as a secondary antibody. Post-staining images were taken with FLUOVIEW10-ASW laser scanning confocal microscope (Olympus, Japan).

\subsection{Western blot analysis}

Cell lysates were subjected to SDS/PAGE on a $4-15 \%$ gradient gel and transferred onto a polyvinylidene fluoride (PVDF) membrane. After blocking with 5\% non-fat dry milk in Tris-buffered saline, the membrane was incubated with primary rabbit monoclonal antibody against P62 protein (Santa Cruz Biotechnology), followed by a secondary horseradish peroxidase (HRP)conjugated anti-rabbit IgG antibody (Santa Cruz Biotechnology). Subsequently, the blot was developed using an ECL detection kit (Thermo, CA, USA).

\section{Results and Discussion}

\subsection{HBV replication induces autophagic vacuole formation}

Autophagy initiates with the formation of phagophore (an isolation membrane). One of the components involved in this early event is the protein $\mathrm{LC} 3$, which becomes lipidated (LC3-II) and associates with the newly formed double-membrane vesicles. It has been shown that exogenous GFP-LC3 (green fluorescence protein conjugated with microtubule-associated protein 1 light chain 3) behaves in a similar fashion as does endogenous LC3, upon autophagy induction. We took advantage of the GFP-LC3 fusion protein, and studied whether continual expression of HBV proteins leads to autophagy induction in HepG2 cells. We transfected the plasmid expressing GFP-LC3 into both HepG2 and HepG2.2.15 cells, and studied the distribution of the GFP signals in the cells through fluorescence microscopy. A diffused distribution of GFP signal was observed in HepG2 cells (Figure 1A) that was consistent with the distribution of the soluble form of LC3 in the absence of autophagy. The GFP signal in HepG2.2.15 cells, however, appeared as punctuated dots much 


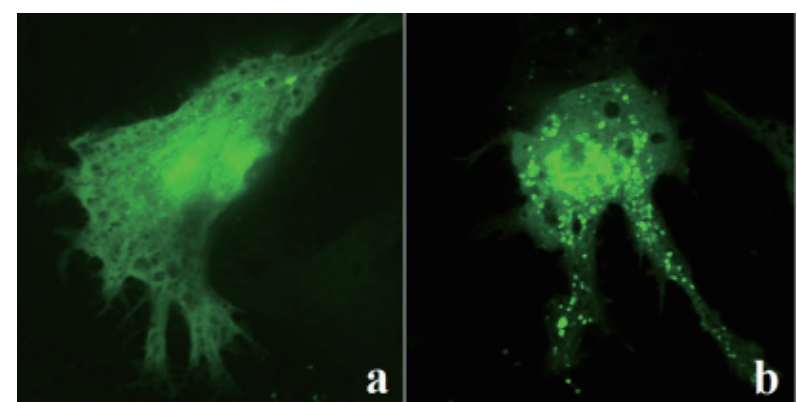

Figure 1. Induction of autophagic vacuoles by HBV. HepG2 cells (panel a) and HepG2.2.15 cells (panel b) were transfected with pEGFP-C1-LC3. Images were taken at $24 \mathrm{~h}$ posttransfection. The Green color was shown in a diffused pattern in HepG2 cells and in punctate dots in HepG2.2.15 cells. The punctate dots represent formation of autophagic vacuoles.

different than what was observed in HepG2 cells (Figure $1 \mathrm{~B})$. The punctuated localization is consistent with the observation that during autophagy the lipidated LC3 is associated with the autophagosomal membrane. The production of HBV by HepG2.2.15 cells was verified by FQ-PCR (data not shown). These observations indicated that the presence of HBV proteins induce autophagy in host cells.

This autophagy can be further augmented by nutrient starvation, one of the many common autophagy-inducing signals. HepG2.2.15 cells expressing GFP-LC3 were cultured in serum-free DMEM medium for $24 \mathrm{~h}$. As shown in Figure 2, the intensity of green fluorescence produced from GFP-LC3 became stronger and more punctuated than those observed in cells cultured under normal conditions (comparing panels a and d). This indicated that nutrient starvation could further induce autophagy process in cells with an already elevated autophagy level due to virus expression.

HBV induced autophagy can be abolished by 3-methyladenine (3-MA), an inhibitor for type III PI3 kinase Vps34. When GFP-LC3 expressing HepG2.2.15 cells were treated with $10 \mathrm{mM}$ 3-MA (Sigma, CA, USA), the distribution of green fluorescence became more diffused than that of the untreated control, suggesting localization of LC3 is more cytosolic (Figure 2 comparing panels $\mathbf{g}$ and $\mathbf{d}$ ).

\subsection{The expression of $H B s A g, H B c A g$, and hepatitis $B$ polymerase and their localization}

We then used immunofluorescence to study whether autophagy is required for the expression of HBV proteins, specifically that of hepatitis B polymerase, $\mathrm{HBcAg}$, and HBsAg.

We did not find significant changes in the expression of hepatitis B polymerase or $\mathrm{HBcAg}$ when autophagy is further enhanced by starvation. The intensities in fluorescence reflecting the abundances of protein were not significantly different in cells growing in normal conditions or starved conditions (Figures 2A and 2B,
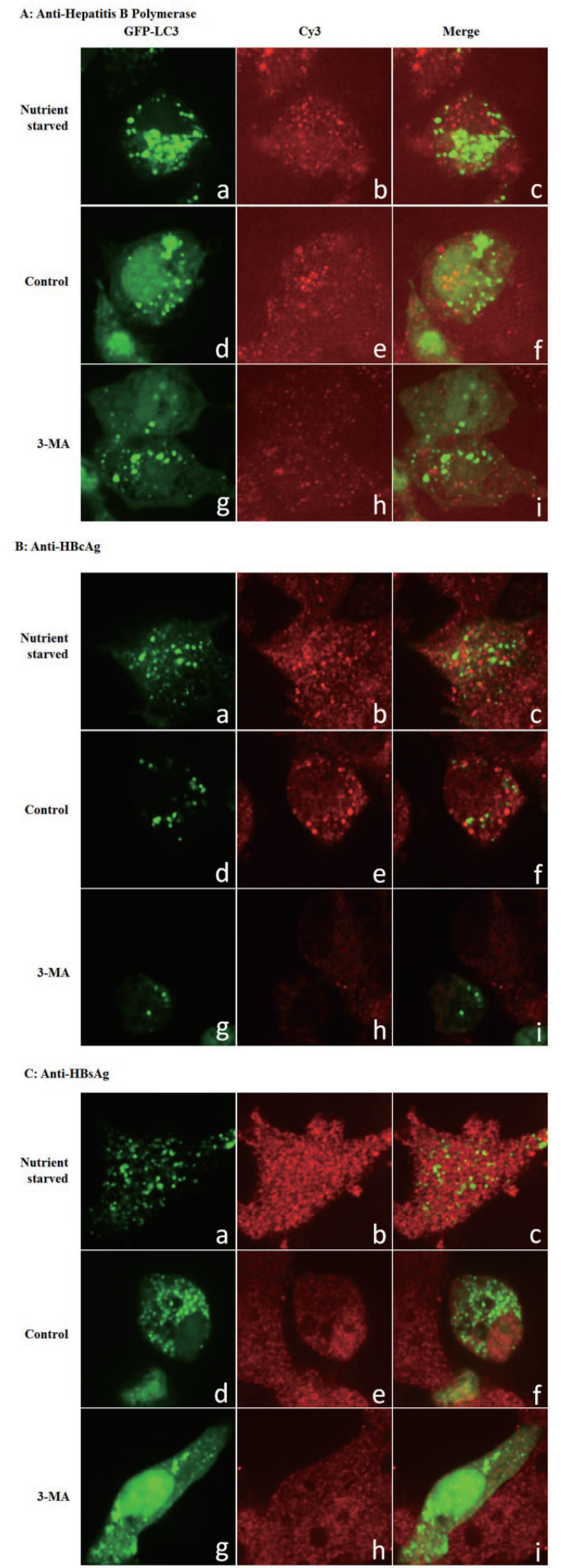

Figure 2. The effect of autophagy on HBV protein expressions. HepG2.2.15 cells transfected with pEGFP-C1LC3 were cultured in serum-free DMEM medium, normal DMEM medium as control, or with 3-MA in DMEM medium. HBV protein expressions were detected with antibodies against hepatitis B polymerase (A), HBcAg (B) or HBsAg (C) and subsequent with the Cy3-conjugated goat anti-mouse secondary antibody. Localization of autophagic vacuoles and HBV was observed by merging panels between GFP-LC3 (green) and Cy3 (red) signals. 


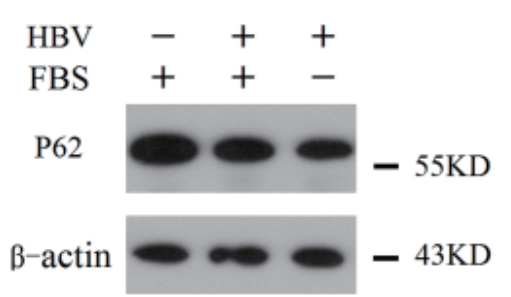

Figure 3. The autophagy induced by HBV leads autophagic degradation. HepG2 cells were cultured in normal DMEM medium (lane 1), while HepG2.2.15 cells were cultured either in normal DMEM medium (lane 2) or serum-free DMEM medium (lane 3). Cells were lysed for Western-blot analysis using the rabbit monoclonal antibody against $\mathrm{P} 62$ protein antibody (top panel). Actin (bottom panel) was also shown as a loading control.

comparing panels $\mathbf{b}$ and $\mathbf{e}$ ). However, the abundances of both proteins reduced in cells where autophagy is inhibited by 3 -MA (Figures $2 \mathrm{~A}$ and $2 \mathrm{~B}$, comparing panels $\mathbf{e}$ and $\mathbf{h}$ ). HBsAg expression seems to be different. We found that the fluorescence intensity of HBsAg is increased during starvation, when autophagy is enhanced in addition to the induction by HBV. Inhibition of autophagy by 3-MA has little effect on its expression (Figure 2C, comparing panel $\mathbf{e}$ and $\mathbf{h}$ ).

It is reported that several human viruses, such as $\mathrm{HCV}$, use autophagic vesicles as membranous support for translation of viral protein and enhancement of viral RNA replication $(12,13,16)$. We investigated whether HBV similarly uses the autophagic vesicles as its location of replication. The merged images between GFP-LC3 and antibody staining for viral proteins, HBV polymerase, HBcAg, or HBsAg showed that the membrane-associated LC3 does not co-localize with viral proteins, suggesting that HBV may not occupy the autophagic vesicles as its location for viral protein translation and DNA replication (Figure 2, panels c, f, and i).

\subsection{The autophagy induced by HBV leads autophagic degradation}

SQSTM1/p62 protein is an ubiquitin-binding scaffold protein that binds directly to LC3 via the LC3 interacting region (LIR), and interacts with ubiquitinated proteins through the C-terminal ubiquitin associated (UBA) domains. SQSTM1/p62 can link ubiquitinated proteins to autophagosome for degradation, and itself can also be degraded through autophagy (17). The cellular levels of SQSTM1/p62 vary with the induction or inhibition of autophagy; it therefore often serves as a marker for autophagic degradation. We studied whether autophagy induced by HBV leads to autophagic degradation by detecting the SQSTM1/p62 protein level with Western blot. Analysis showed that SQSTM1/p62 protein level was decreased in HepG2.2.15 cells comparing to HepG2 cells, and it was further reduced in HepG2.2.15 cells cultured in serum-free DMEM medium (Figure 3).
This indicated that autophagy induced by HBV leads to autophagic degradation.

Autophagy is a protective process that recycles damaged organelles and unwanted proteins to ensure cell viability under starvation and stress conditions. Increasing amounts of evidence has shown that it is also involved in regulating innate and adaptive immunity. It is particularly important in the cellular defense against intracellular pathogens, in which xenophagy recognizes intracellular microbes and targets them to the autophagy pathway for degradation. Thus, many pathogens, including viruses, have developed strategies for self-protection and further manipulate or utilize autophagy to their own advantages. For example, Epstein-Barr virus (EBV) and Kaposi's Sarcoma-Associated Herpes Virus (KSHV) negatively regulate autophagy during latency, whereas poliovirus $(18,19)$, porcine reproductive and respiratory syndrome virus (PRRSV) (20), encephalomyocarditis virus (21), and picornavirus (22), utilize components of the autophagy pathway to promote viral replication. Some of the viruses use the autophagosome as a membrane structure to support its replication as well as to hijack it as a mechanism for releasing the packaged virions in a non-lytic manner. This promotes the fusion of the autophagosome with the plasma membrane $(12,19,21,23,24)$. While viruses promote autophagy induction, it has been shown that they have developed mechanisms to inhibit the maturation of autophagosomes by preventing the fusion between the autophagosome and the lysosome $(19,20)$. However, recent findings also indicate that some RNA viruses benefit from autophagosome maturation. For example, acidification of the autophagasome is required for poliovirus particle assembly and virion maturation (18).

Our study showed that autophagy could be induced in HepG2 cells when the HBV replication became persistent in the cells, and this autophagy could be further enhanced by nutrient starvation. This is consistent with the previous findings that HBV induces autophagy in host cells via its HBx protein (14). Several studies showed that HBx interacts with C3-PI3K to initiate the autophagy process and the induction of autophagy is required for HBV DNA replication in vitro (14). In vivo, blocking autophagy by knocking out Atg5 greatly reduced HBV DNA replication (25). Our data also suggested that the induction of autophagy was necessary for $\mathrm{HBV}$ gene expression. The expressions of $\mathrm{HBcAg}$ and Hepatitis DNA polymerase were reduced when the autophagy was blocked by 3-MA, although they were not further increased when the enhanced autophagy was induced by starvation in addition to HBV. However, the expression of HBsAg was a little different. We found that inhibition of autophagy by 3-MA had little effect on the expression of HBsAg, whereas starvation led to the increase of HBsAg expression. It is not clear why the modulation of HBsAg expression is different. As 
it is known that HBsAg expressions in active hepatitis patients were found in a few discrete hepatocytes with a diffuse cytoplasmic distribution, it is possible that HBsAg expression is not directly related to viral replication cycle (26).

Unlike several RNA viruses like HCV, HBV does not appear to replicate in autophagosome. We found that viral proteins, such as $\mathrm{HBcAg}$, hepatitis B polymerase, or HBsAg did not localize in the autophagosome; i.e. there was no co-localization between viral proteins and autophagosome marker LC3. This observation suggests that HBV may not replicate and assemble in the autophagosome.

In contrast to previous observations, we found that SQSTM1/p62 level was reduced in HepG2.2.15 cells, and was further reduced in cells cultured in starvation condition $(14,27)$. Our data indicated that the fusion of autophagosome with lysosome was not blocked in HBV infected cells. Part of the reason for this discrepancy may possibly due to the use of different hepatoma cell lines in our study. It is also possible, particularly in this case that HBV does not need use autophagosome as replication location, that there might be certain mechanisms that virus can selectively block protein degradation in the autophagosome based on its own needs.

This study reconfirmed several previous findings but the exact role of autophagy in HBV life cycle is still not clear. Sir et al. reported that autophagy could be induced by $\mathrm{HBx}$ protein and was required for efficient viral DNA replication; however, blocking the fusion of autophagosome and lysosome lead to reduced level of viral RNA but had little effect on viral DNA production (14). Li et al. showed that autophagy was needed for enveloped virion production at the ER, but had only slight effect on HBV DNA replication (27). Lazar $\mathrm{C}$ et al. reported that ER degradation-enhancing, mannosidase-like proteins (EDEM) were up-regulated in HepG2.2.15 cells, and the presence of viral surface proteins and EDEM1 led to the degradation of envelope proteins through autophagy. They suggested that it might be the mechanism for HBV to control the level of virions produced in infected cells and to establish chronic infection (28).

In summary, our study suggested that autophagy was induced by HBV, and was required for the viral proteins $\mathrm{HBcAg}$ and hepatitis $\mathrm{B}$ polymerase expression. However, HBV did not block the protein degradation through an autophagy pathway, and neither did it use the autophagosome as its site for DNA replication or virion assembly. Whether the protein degradation through autophagy pathway plays any role in facilitating the HBV life cycle needs further investigation.

\section{Acknowledgements}

We thank Xiangyang Liu for providing medical writing services. This study was supported by National Key
Technology Research and Development Program of China 2012 (grant number BAI06B01), National Natural Science Foundation of China (81201566), China Medical Board of New York (CMB) (11-045), and the Wu Jieping Medical Foundation (LDWMF-SY-2011B002).

\section{References}

1. Su IJ, Hsieh WC, Tsai HW, Wu HC. Chemoprevention and novel therapy for hepatocellular carcinoma associated with chronic hepatitis B virus infection. Hepatobiliary Surg Nutr. 2013; 2:37-39.

2. El-Serag HB. Epidemiology of viral hepatitis and hepatocellular carcinoma. Gastroenterology. 2012; 142:1264-1273 e1.

3. Qi FH, Wang ZX, Cai PP, Zhao L, Gao JJ, Kokudo N, Li AY, Han JQ, Tang W.Traditional Chinese medicine and related active compounds: A review of their role on hepatitis B virus infection. Drug Discov Ther. 2013; 7:212-224.

4. Miao R, Luo H, Zhou H, et al. Identification of prognostic biomarkers in hepatitis B virus-related hepatocellular carcinoma and stratification by integrative multi-omics analysis. J Hepatol. 2014; 61:840-849.

5. Huang J, Brumell JH. Autophagy in immunity against intracellular bacteria. Curr Top Microbiol Immunol. 2009; 335:189-215.

6. Gutierrez MG, Master SS, Singh SB, Taylor GA, Colombo MI, Deretic V. Autophagy is a defense mechanism inhibiting BCG and Mycobacterium tuberculosis survival in infected macrophages. Cell. 2004; 119:753-766.

7. Nakagawa I, Amano A, Mizushima N, Yamamoto A, Yamaguchi H, Kamimoto T, Nara A, Funao J, Nakata M, Tsuda K, Hamada S, Yoshimori T. Autophagy defends cells against invading group A Streptococcus. Science. 2004; 306:1037-1040.

8. Cao X, Liu B, Cao W, Zhang W, Zhang F, Zhao H, Meng R, Zhang L, Niu R, Hao X, Zhang B. Autophagy inhibition enhances apigenin-induced apoptosis in human breast cancer cells. Chin J Cancer Res. 2013; 25:212222.

9. Facciorusso A, Antonino M, Del Prete V, Neve V, Scavo MP, Barone M. Are hematopoietic stem cells involved in hepatocarcinogenesis? Hepatobiliary Surg Nutr. 2014; 3:199-206.

10. Schmid D, Munz C. Innate and adaptive immunity through autophagy. Immunity. 2007; 27:11-21.

11. Kirkegaard K, Taylor MP, Jackson WT. Cellular autophagy: Surrender, avoidance and subversion by microorganisms. Nat Rev Microbiol. 2004; 2:301-314.

12. Jackson WT, Giddings TH, Jr., Taylor MP, Mulinyawe S, Rabinovitch M, Kopito RR, Kirkegaard K. Subversion of cellular autophagosomal machinery by RNA viruses. PLoS Biol. 2005; 3:e156.

13. Prentice E, Jerome WG, Yoshimori T, Mizushima N, Denison MR. Coronavirus replication complex formation utilizes components of cellular autophagy. J Biol Chem. 2004; 279:10136-10141.

14. Sir D, Tian Y, Chen WL, Ann DK, Yen TS, Ou JH. The early autophagic pathway is activated by hepatitis B virus and required for viral DNA replication. Proc Natl Acad Sci U S A. 2010; 107:4383-4388.

15. Tang H, Da L, Mao Y, Li Y, Li D, Xu Z, Li F, Wang Y, 
Tiollais P, Li T, Zhao M. Hepatitis B virus X protein sensitizes cells to starvation-induced autophagy via upregulation of beclin 1 expression. Hepatology. 2009; 49:60-71.

16. Suhy DA, Giddings TH, Jr., Kirkegaard K. Remodeling the endoplasmic reticulum by poliovirus infection and by individual viral proteins: An autophagy-like origin for virus-induced vesicles. J Virol. 2000; 74:8953-8965.

17. Moscat J, Diaz-Meco MT. p62 at the crossroads of autophagy, apoptosis, and cancer. Cell. 2009; 137:10011004.

18. Richards AL, Jackson WT. Intracellular vesicle acidification promotes maturation of infectious poliovirus particles. PLoS Pathog. 2012; 8:e1003046.

19. Taylor MP, Jackson WT. Viruses and arrested autophagosome development. Autophagy. 2009; 5:870871.

20. Sun MX, Huang L, Wang R, Yu YL, Li C, Li PP, Hu XC, Hao HP, Ishag HA, Mao X. Porcine reproductive and respiratory syndrome virus induces autophagy to promote virus replication. Autophagy. 2012; 8:14341447.

21. Zhang Y, Li Z, Ge X, Guo X, Yang H. Autophagy promotes the replication of encephalomyocarditis virus in host cells. Autophagy. 2011; 7:613-628.

22. Klein KA, Jackson WT. Picornavirus subversion of the autophagy pathway. Viruses. 2011; 3:1549-1561.

23. Le Sage V, Banfield BW. Dysregulation of autophagy in murine fibroblasts resistant to HSV-1 infection. PLoS One. 2012; 7:e42636.

24. Weiskirchen R, Tacke F. Cellular and molecular functions of hepatic stellate cells in inflammatory responses and liver immunology. Hepatobiliary Surg Nutr. 2014; 3:344363.

25. Tian Y, Sir D, Kuo CF, Ann DK, Ou JH. Autophagy required for hepatitis $\mathrm{B}$ virus replication in transgenic mice. J Virol. 2011; 85:13453-13456.

26. Nakopoulou L, Adraskelas N, Stefanaki K, Zacharoulis D, Hadziyannis S. Expression of HBsAg and $\mathrm{HBcAg}$ in liver tissue: Correlation with disease activity. Histol Histopathol. 1992; 7:493-499.

27. Li J, Liu Y, Wang Z, Liu K, Wang Y, Liu J, Ding H, Yuan Z. Subversion of cellular autophagy machinery by hepatitis B virus for viral envelopment. J Virol. 2011; 85:6319-6333.

28. Lazar C, Macovei A, Petrescu S, Branza-Nichita N. Activation of ERAD pathway by human hepatitis B virus modulates viral and subviral particle production. PLoS One. 2012; 7:e34169.

(Received April 2, 2015; Revised April 8, 2015; Accepted April 20, 2015) 